\title{
Patterns, Movement and Clinical Diagnosis of Abdominal Adhesions
}

\author{
Benjamin Wright $^{1, \star}$, John Fenner ${ }^{1}$, Richard Gillott ${ }^{2}$, \\ Paul Spencer ${ }^{2}$, Patricia Lawford ${ }^{1}$, and Karna Dev Bardhan ${ }^{2}$ \\ 1 University of Sheffield, UK \\ 2 Rotherham General Hospital, UK
}

\begin{abstract}
Patterns in normal abdominal movement captured with medical imaging can be recognised by a trained radiologist but the process is time consuming. Abdominal adhesions present a diagnostic problem in which the radiologist is asked to detect abnormal movement that may be indicative of pathology. This paper postulates that the use of image analysis can augment the diagnostic abilities of the radiologist in respect of adhesions. Proof of concept experiments were conducted insilico to explore the effectiveness of the technique. The results indicate that trained participants are accurate in their assessment of abnormalities when supplied with additional information from image analysis techniques. However without the additional information, participants made incorrect diagnoses on many occasions. ROC methods were used to quantify the outcomes of the in-silico experiment.
\end{abstract}

Keywords: Pattern recognition, Image analysis, Abdominal Adhesions, In-silico modelling.

\section{Introduction}

Abdominal adhesions are fibrous bands of connecting tissue that can result from injury to the abdominal contents [1]. Often a direct result of mechanical injury during surgery [2], they adhere anatomical components to one-another or to the abdominal wall and as a result can inhibit the normal function of the abdomen [34. Adhesions often lie unnoticed, however, when symptoms present they are frequently diagnosed only through a process of exclusion of other, more common, disorders [5]. They are structurally similar to surrounding tissues and are often volumetrically insubstantial, producing insufficient signal for direct detection using non-invasive medical imaging. However, it is a premise of this work that imaging can be used for diagnosis by identifying patterns of movement that are characteristic of adhesions. This is supported by the work of other groups that promote the use of Magnetic Resonance Imaging (MRI) 6]7] or Ultrasound Scanning (US) [8] as an effective means of non-invasive diagnosis. Both techniques

\footnotetext{
* The authors would like to thank the Bardhan Research and Education Trust of Rotherham (BRET) and the Engineering and Physical Sciences Research Council (EPSRC) for their financial support of this work.
} 
have advantages but this paper focuses on MRI as the technique of choice and discusses image processing based data reduction techniques to aid the pattern recognition task associated with identifying signatures of disturbed movement caused by adhesions.

\subsection{Signatures Observed Using MRI}

The most effective protocol for non-invasive diagnosis of adhesions requires that a trained radiologist monitors the movement of the abdominal contents as captured by digital imaging 9. This is performed using a series of 2-dimensional cine MRI acquisitions. Each cine MRI is a collection of approximately 15 time-sequential 2-dimensional planar images that cover the respiratory cycle of the patient. To examine the whole abdominal cavity, approximately 15 sagittal and transverse planes are acquired. A total of 30 cine MRI scans comprising of more than 450 individual 2-dimensional MRI scans are then presented to the radiologist. The radiologist observes the movement of the abdominal contents throughout the cine sequences in an attempt to detect any abnormal patterns of movement. This is a process that is very time consuming and raises issues about reproducibility.

\subsection{Image Analysis}

This work postulates that image analysis techniques can be used to augment the aforementioned diagnostic procedures. Movement can be quantified using image registration techniques, so that images in the cine sequence are registered to other temporally consecutive images. A gradient-based registration method matches an image pair and produces a vector map of the transformation required to move from one to the other [10]. The vector map is a continuous field that describes the mapping of structures from one image to equivalent structures in the second image and minimises a cost function appropriate to the medical imaging modality. The vector map can subsequently be analysed and visualised in forms that promote recognition of characteristic signatures, which may be indicative of adhesion induced movement disturbances.

Hypothesis. The use of appropriate image analysis methods can augment the diagnostic efficacy of the radiologist through reduction of the pattern recognition task.

\section{Method}

The hypothesis was examined using in-silico models in which a virtual representation of the diagnostic challenge was trialled with numerous observers. The model, which is based on a 2-dimensional finite element structural system, comprises of 4-node square elements and is complemented with synthesised images that were derived from anatomical features. Each structural element featured the same base stiffness, but was perturbed with additional noise of up to $\pm 30 \%$. The structural nature of the mesh enabled forces to be applied at the nodes to 


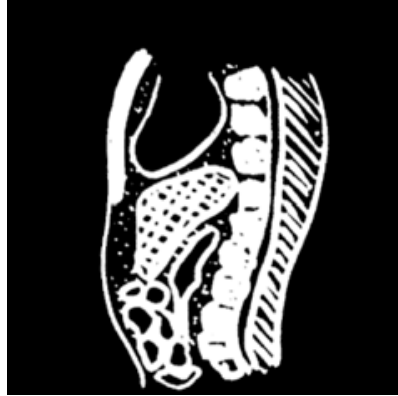

(a) Rest

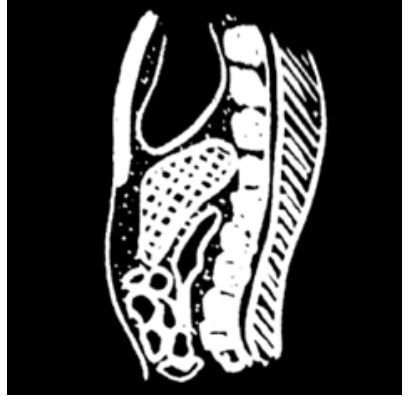

(b) Stretched

Fig. 1. In-silico images. (a) The rest image that is added to the finite element mesh. (b) Maximum force has been applied to the mesh, creating the stretched image.

produce distortions. This facilitated crude representation of abdominal movement, subject to the physiological forces experienced from diaphragmatic movement. An image (Fig. 11) was laid over the finite element mesh and image pixel co-ordinates computed relative to each element. When subjected to specified forces, the mesh distortions were computed and interpolation techniques were used to calculate and redraw a consistent distorted image. By changing the strength of the forces applied to the system, multiple distortions were created and the resulting sequence of 20 images collated into a cine sequence. The movement of the image over time was analogous to images generated in the clinical setting. The design of the model permits modification of the stiffness of particular elements and therefore is capable of introducing anomalous movement and disturbances. The task of identifying stiff elements from features in the moving image was intended to reflect the clinical task of diagnosing abdominal movement disturbances caused by the presence of adhesions and was considered by consultant radiologists to be suitably challenging.

\subsection{Image Analysis}

Image registration methods were used to compare temporally consecutive images from the in-silico cine sequence. Registration involves the mapping of positions in a source image to their relative positions in a target image. This is an iterative process that involves transforming the source image and measuring the difference between the transformed and the target image. The process continues until an acceptable match is found and an appropriate cost function for the registration minimised. The Sheffield Image Registration Toolkit (ShIRT) was used in this work to perform the registration [11. Once registration is complete, a vector mapping indicating the required transformation can be analysed. Image registration requires that the difference between the images is small enough for correct estimation of the required transformation. Subsequent analysis of the vector mapping is used to present the data. In this work, the magnitude of the 
vectors and the local gradients of the vector magnitudes were considered to be appropriate indicators of movement. Colour scales were used to present the data.

\subsection{Isis}

These elements were encapsulated in a software package called Isis (In-Silico Investigation System), created to test the effectiveness of the technique. It was specifically designed to present a participant with a cine sequence that randomly featured the presence/absence of an adhesion and asked them to diagnose the condition as presented. This included recording the certainty of diagnosis and was performed with and without the additional information provided by the image analysis.

Before conducting the tests, each participant was required to take part in a short training programme. The software for the training was bundled with Isis and featured a similar user interface. Two modes were available to the participant, the first of which was a demonstration mode. In this mode the participant was presented with two cine data sets; one of these featured an adhesion and the other did not. The participant was told which was which and was able to study the differences in both the cine data and the additional vector information provided by the image analysis. Once the participant was satisfied with their ability to detect the presence of an adhesion, the second training mode could

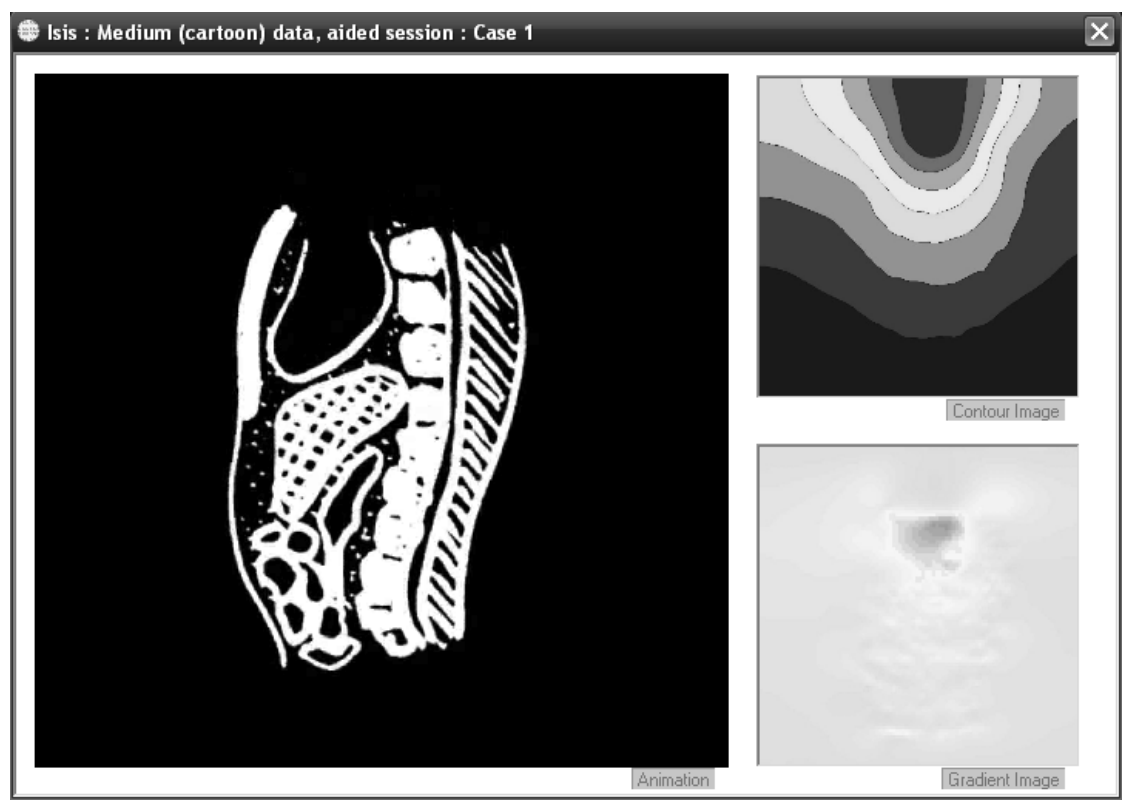

Fig. 2. Isis graphical user interface. Cine data shown in the left hand pane of the window. Optional information provided by image analysis features on the right hand side. The top right pane shows a contour plot of vector magnitude and the bottom right pane shows a gradient based image. 
be used. In this mode the participant was presented with a random cine data set. Their challenge was to be able to identify the presence of an adhesion with adequate certainty and consistency. Successful diagnoses at this stage enabled the participant to progress to the main Isis application for quantification of their diagnostic performance.

\subsection{Test Protocol}

To evaluate the effectiveness of the technique, Isis was used to educate and examine two medically trained participants with 80 cine data sets in a randomised order. Similar to the ratio experienced by radiologists, half of the data sets featured an adhesion and the other half did not. In order to maintain simplicity in the test, the adhesion was always in the same location if present. Each participant was asked to diagnose the presence/absence of an adhesion in all cases with and without the additional information provided by the image processing. When making a diagnosis the participant was asked to quantify their certainty of diagnosis using a subjective four-level scale featuring the levels: Pure guess, Possibly, Probably and Definitely. Isis recorded the participants response, their time taken to respond and the level of certainty. The information was recorded to an output file and later examined using Receiver Operating Characteristic (ROC) methods [12].

\section{Results}

Isis scores as recorded for the two participants were analysed as described above. The resulting data points were plotted in an ROC space. Data was also available on the time taken for the user to make a decision and their certainty when making it.

\subsection{ROC Data}

ROC methods were employed to evaluate the effectiveness of the additional information, provided by the image analysis, available to the participant. Total numbers of true positives (TP), false positives (FP), true negatives (TN) and false negatives (FN) were calculated using the data provided by Isis. The true positive and false positive rates (TPR and FPR respectively) were calculated using the following standard formulae 12 .

$$
\begin{aligned}
& T P R=\frac{T P}{T P+F N} \\
& F P R=\frac{F P}{F P+T N}
\end{aligned}
$$

Figures 3 and 4 display the corresponding ROC curves which plot TPR against FPR for the different decision thresholds recorded in the test. Where appropriate, 


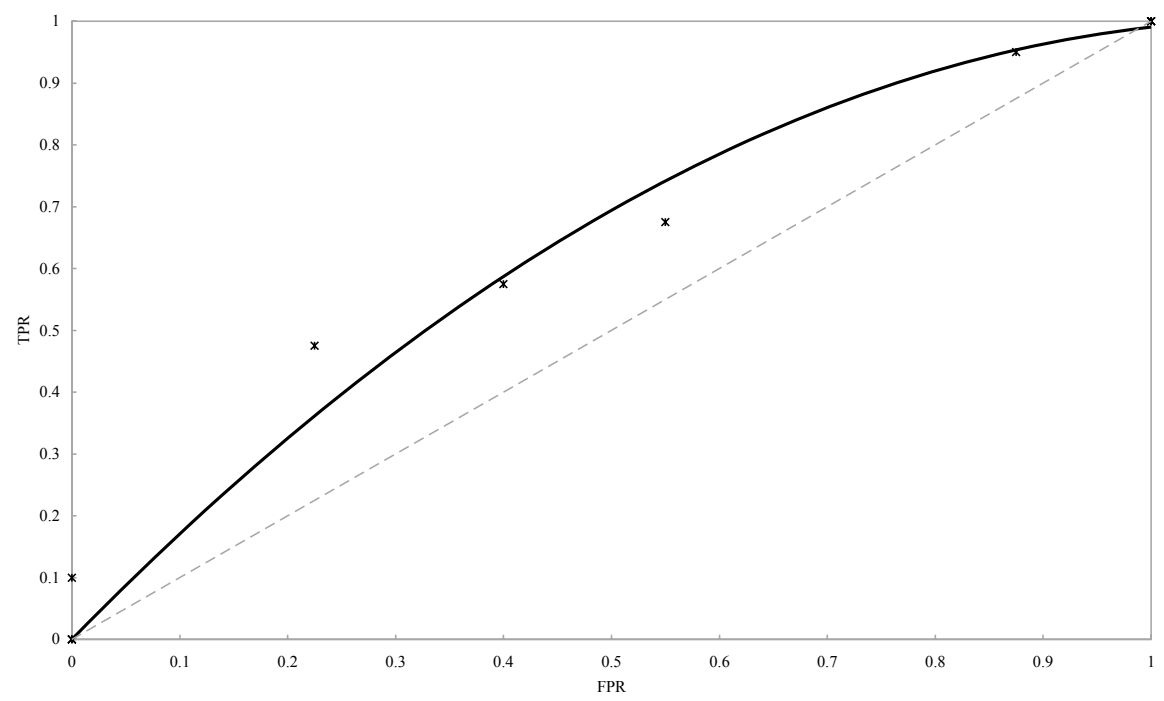

(a) Without additional information

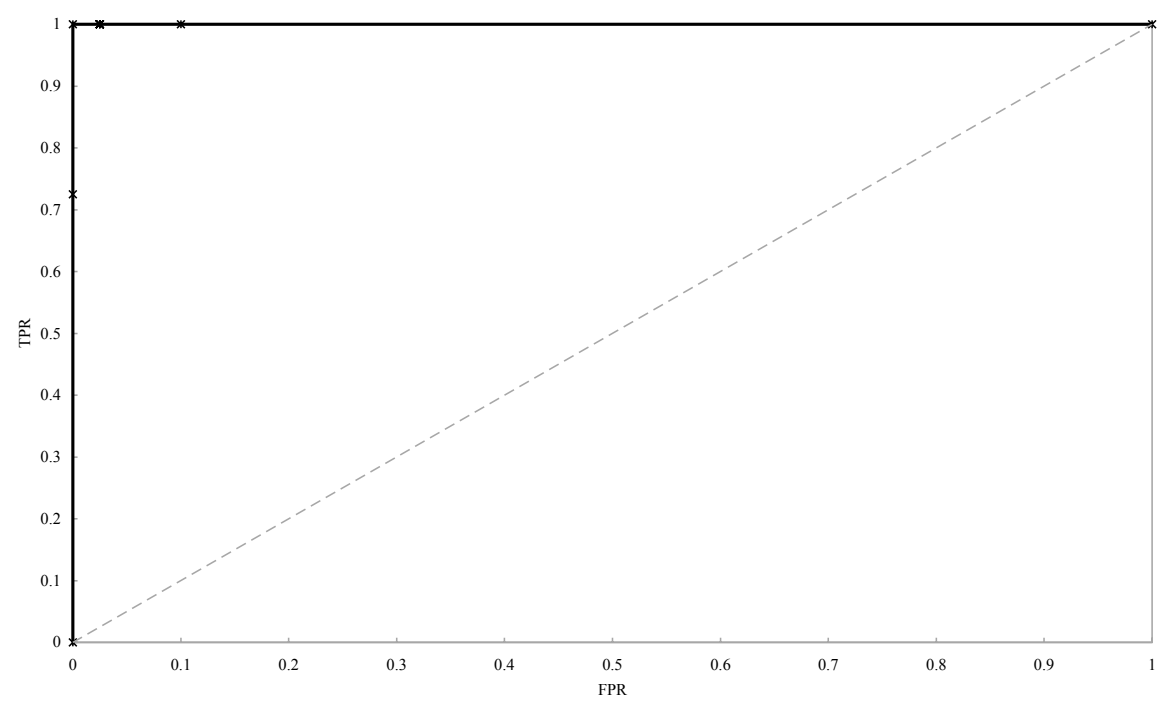

(b) With additional information

Fig. 3. ROC Data for Participant 1 gathered by Isis, where TPR = true positive rate and FPR $=$ false positive rate. Dashed line represents the line of no dicrimination. Data is presented for the test without and with additional image processing information, (a) and (b) respectively. Polynomial best fit line shown for Unaided data. Area under the ROC curves are 0.63 and 1.00 respectively. 


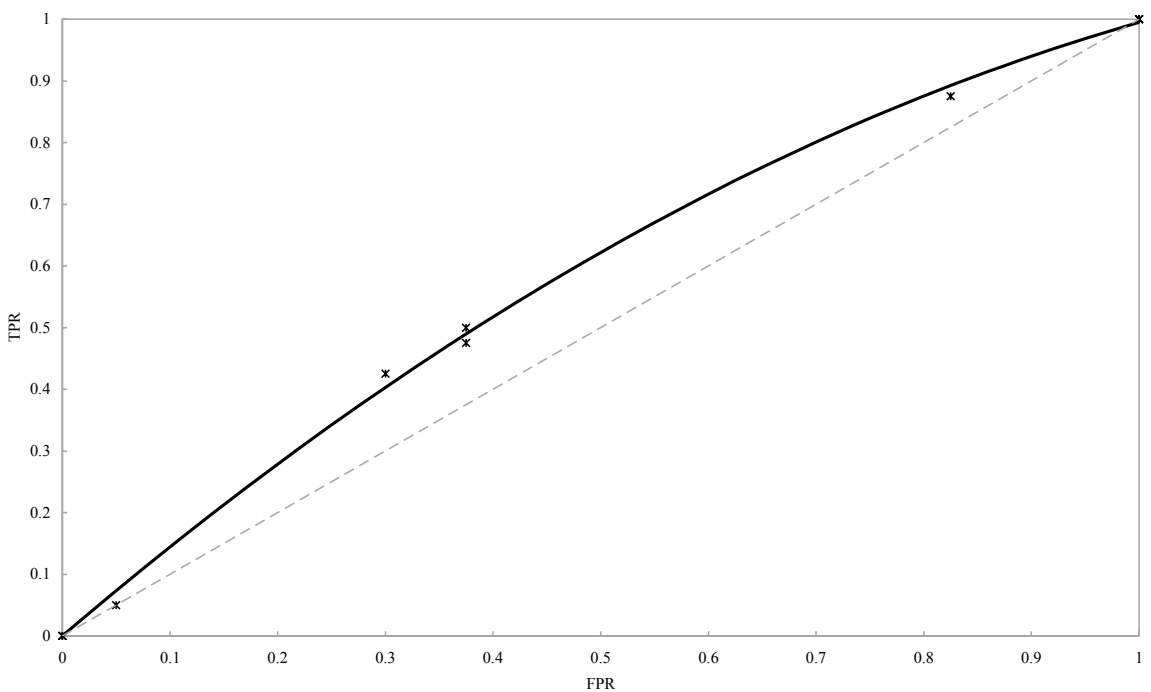

(a) Without additional information

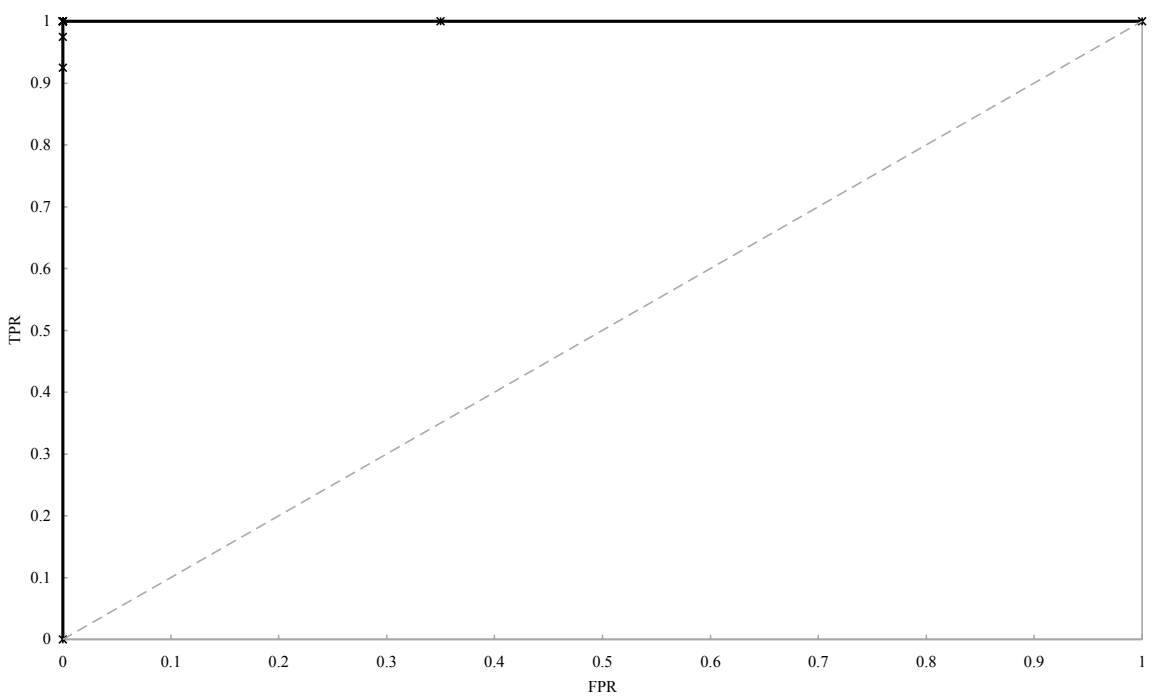

(b) With additional information

Fig. 4. ROC Data for Participant 2 gathered by Isis, where TPR = true positive rate and FPR $=$ false positive rate. Dashed line represents the line of no dicrimination. Data is presented for the test without and with additional image processing information, (a) and (b) respectively. Polynomial best fit line shown for Unaided data. Area under the ROC curves are 0.58 and 1.00 respectively. 
a second order polynomial line of best fit has been added. The results show that, rather surprisingly, with the additional information provided by the image analysis both participants were able to obtain perfect scores when diagnosing the presence of an adhesion. Both curves display the line of no discrimination and it is seen that all results lie above this.

\subsection{Data Tables}

The Isis output file contains information regarding the participants certainty in their choice of diagnosis and the time taken to make a decision. This information is displayed in tables 1 and 2 .

Table 1. Showing the number of times a diagnosis was made with each level of certainty

\begin{tabular}{c|cc|cc} 
& \multicolumn{2}{c}{ Participant 1 } & \multicolumn{2}{c}{ Participant 2 } \\
& \multicolumn{2}{c}{ Image analysis status } & \multicolumn{2}{c}{ Image analysis status } \\
Level of certainty & Unavailable & Available & Unavailable & Available \\
\hline Pure guess & 21 & 0 & 6 & 0 \\
Possibly & 48 & 1 & 58 & 1 \\
Probably & 11 & 14 & 16 & 16 \\
Definitely & 0 & 65 & 0 & 63
\end{tabular}

Table 2. Showing the average time taken to make a diagnosis

\begin{tabular}{c|cc|cc} 
& \multicolumn{2}{c}{ Participant 1 } & \multicolumn{2}{c}{ Participant 2 } \\
Tmage analysis status & \multicolumn{2}{c}{ Image analysis status } \\
Time (sec) & Unavailable & Available & Unavailable & Available \\
\hline Average time taken & $5.6 \pm 0.5$ & $1.5 \pm 0.5$ & $10.8 \pm 0.5$ & $2.5 \pm 0.5$
\end{tabular}

\section{Discussion}

The data confirms the supposition of the hypothesis that the use of appropriate image analysis can augment the diagnostic efficacy of the radiologist through reduction of the pattern recognition task. Participants not supported with image analysis are consistently poor in their diagnosis. In-silico results imply that the technique may have potential to be applied to clinical data where occurrences of adhesions are common after surgery. Radiological methods that allow noninvasive diagnosis of the condition present a difficult and time consuming pattern recognition challenge and this has been replicated in-silico. Furthermore, image analysis has demonstrated the ability to reduce this task through registration of temporally consecutive frames and subsequent presentation of the registration information. 


\subsection{In-Silico Model Critique}

The clinical methodology and its augmentation by image processing has been evaluated using in-silico models designed to capture the essence of free and disturbed abdominal motion during respiration. The motion observed in the model achieves this but its simplicity and structural foundation is associated with numerous limitations. Unlike the abdomen, the model does not currently allow for structures to slide past one another [14. Instead they are connected by elements that can be made flexible but offer no opportunity for slide. This limits interpretation in the context of clinical images, but nonetheless encapsulates the essence of diagnosing an underlying structural abnormality by observing disturbances to normal movement. The images for this exercise were based on preliminary tests that indicated, in the absence of image processing support, the participant found it increasingly difficult to identify anomalous areas of raised stiffness as the complexity of the image increased. The chosen image was a compromise; being simple enough to offer the participant the best possible chance of detecting a disturbance and yet providing an image with sufficient information content for the image processing algorithm to operate effectively [11].

The structural model can be enhanced beyond its current state. Variables such as the shape of elements and individual stiffness can be altered to make the system bear a closer resemblance to the clinical anatomy. The planar nature of clinical MRI means that sometimes 3-dimensional anatomical structures can move in and out of the imaging plane. The radiologist recognises these occurrences and can cross planes to track anatomical structures. This is something that should be addressed for future in-silico models.

Forces applied to the model were simple and linear unlike the forces seen in the abdomen. This produced consistent distortions in the image and made the pattern recognition task easier when considering the image analysis visualisations.

\subsection{Experimental Results}

Isis was used to evaluate the effectiveness of the proposed diagnostic augmentations. Participants in the test were asked to operate the software which would record their diagnosis of the presence/absence of an adhesion. The test was completed with and without the additional information provided by the image analysis. As seen from the results, the additional information significantly enhanced the diagnostic capability of the participant. The area under the ROC curve is an accepted measurement that quantifies the effectiveness of diagnosis [12. The area is measured as 1 if the operator is always correct and 0 if they are never correct, an area of 0.5 is equivalent to random guessing and represented by the line of no discrimination. Participant 1 had an area under the curve of 0.63 when not provided with image analysis data. When provided with the additional information, they were able to achieve a perfect score of 1.00 meaning their diagnosis of anomalous stiffness, based on image movement, was always correct. Participant 2 had an area under the curve of 0.58 when not provided with additional information and an area of 1.00 when provided with the image analysis. 
The perfect scores for both participants when provided with additional information from image analysis might indicate that the diagnostic challenge was too easy, but the score recorded when not provided with this information does not support this. Both participants recorded scores that deviated slightly from the line of no discrimination indicating some success in diagnosis without the additional information. This is supported by the data in table 1 which shows that when provided with additional information from image analysis both participants were much more certain of their diagnosis. Furthermore, when account is taken of the time for a diagnosis to be made, the participants were much quicker when diagnosis was supported with image analysis information.

\subsection{Technique}

The experimental results confirm the hypothesis in this synthetic evaluation. However, it is clear that the in-silico experiment is far removed from the clinical setting. Nonetheless radiologists were consulted throughout the study and from concept to experimental procedure they confirmed that it resonated with the clinical setting. This included the clinical challenge of analysing a vast data set and concerns about reproducibility when additional information from image analysis is absent. When exposed to the software, the radiologists displayed similar results to the medical trainees who made a diagnosis on a total of 160 animations and who also demonstrated misdiagnosis with a degree of confidence; they convinced themselves of distorting movements when in fact there were none. In a clinical context such misdiagnosis could have serious implications for unnecessary surgery and adhesiolysis.

In this simulation, the in-silico adhesion was always in the same place if present. Arguably this is similar to congenitally formed adhesions that attach the liver to the diaphragm for example. Radiologists are often presented with a much more widespread and diffuse diagnostic challenge 9]. This could be replicated in the current in-silico model by randomly placing the stiff element elsewhere within the image. However, the clinical problem also presents adhesions in different forms which will precipitate different movement patterns. Again the current model could be adapted to accommodate for this through modification of the underlying finite element mesh and its flexibility.

\subsection{Clinical Application}

In essence the image analysis methods explored in this paper are data reduction techniques. Vast amounts of complex visual data in the form of cine sequences have been reduced to simpler 2-dimensional plots. This is a typical strategy of pattern recognition solutions and frequently include additional techniques designed to characterise the image under investigation. The complexity of underlying movement disturbances hidden within MRI radiological image (Fig. 5) sequences makes this a very demanding task for any pattern recognition software; but the technique as presented here separates the challenge by isolating data reduction from explicit feature extraction. It is easy for the complex moving 


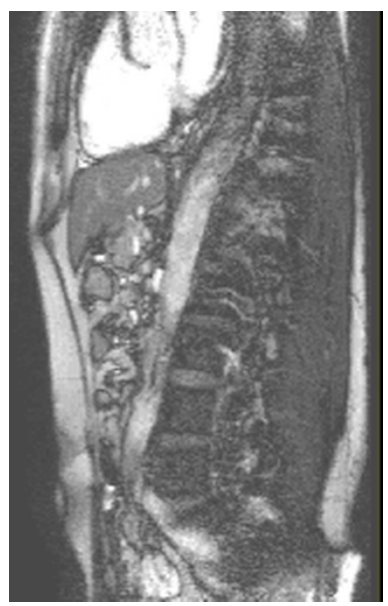

Fig. 5. Contrast enhanced clinical MRI image. Sagittal slice through volunteer showing abdominal contents.

images in the clinical setting to overwhelm the observer but this is dramatically simplified with the data reduction of the image processing which leaves the feature extraction and final classification to the trained eye of the radiologist. This technique does not attempt to completely automate detection of adhesions, but offers to guide the observer to movement anomalies in support of diagnosis.

\section{Conclusion}

This paper has described a simulation scenario that encapsulates important elements associated with clinical diagnosis of adhesions. The method captures the challenge of identifying anomalous movement within a background of complex, normal motion. The application of image processing support has demonstrated the effectiveness of such a tool in guiding the observer to a correct diagnosis. The technique offers hope for the diagnosis of clinical adhesions by similar methods. It confirms the hypothesis that the use of appropriate image analysis methods can augment the diagnostic efficacy of the radiologist through simplification of the pattern recognition task. Further work to improve the realism of the models is ongoing.

\section{References}

1. Boland, G.M., Weigel, R.J.: Formation and prevention of postoperative abdominal adhesions. J. Surg. Res. 132(1), 3-12 (2006)

2. Vrijland, W.W., Jeekel, J., van Geldorp, H.J., Swank, D.J., Bonjer, H.J.: Abdominal Adhesions: Intestinal obstruction, pain, and infertility. Surg. Endosc. 17(7), 1017-1022 (2003) 
3. Cheong, Y.C., Laird, S.M., Li, T.C., Shelton, J.B., Ledger, W.L., Cooke, I.D.: Peritoneal healing and adhesion formation/reformation. Hum. Reprod. Update 7(6), 556-566 (2001)

4. Diamond, M.P., Freeman, L.M.: Clinical implications of postsurgical adhesions. Hum. Reprod. Update 7(6), 567-576 (2001)

5. Swank, D.J., Swank-Bordewijk, S.C., Hop, W.C., van Erp, W.F., Janssen, I.M., Bonjer, H.J., Jeekel: Laparoscopic adhesiolysis in patients with chronic abdominal pain: a blinded randomised controlled multi-centre trial. Lancet 361(9365), $1247-$ $1251(2003)$

6. Katayama, M., Masui, T., Kobayashi, S., Ito, T., Sakahara, H., Nozaki, A., Kabasawa, H.: Evaluation of pelvic adhesions using multiphase and multislice MR imaging with kinematic display. Am. J. Roentgenology 177(1), 107-110 (2001)

7. Lang, R.A., Buhmann, S., Hopman, A., Steitz, H.O., Lienemann, A., Reiser, M.F., Jauch, K.W., Huttl, T.P.: Cine-MRI detection of intraabdominal adhesions: correlation with intraoperative findings in 89 consecutive cases. Surg. Endosc. 22(11), 2455-2461 (2008)

8. Caprini, J.A., Arcelus, J.A., Swanson, J., Coats, R., Hoffman, K., Brosnan, J.J., Blattner, S.: The ultrasonic localization of abdominal wall adhesions. Surg. Endosc. 9(3), 283-285 (1995)

9. Mussack, T., Fischer, T., Ladurner, R., Gangkofer, A., Bensler, S., Hallfeldt, K.K., Reiser, M., Lienemann, A.: Cine magnetic resonance imaging vs high-resolution ultrasonography for detection of adhesions after laparoscopic and open incisional hernia repair: a matched pair pilot analysis. Surg. Endosc. 19(12), 1538-1543 (2005)

10. Crum, W.R., Hartkens, T., Hill, D.L.: Non-rigid image registration: theory and practice. Br. J. Radiol. 77(2), S140-S153 (2004)

11. Barber, D.C., Hose, D.R.: Automatic segmentation of medical images using image registration: diagnostic and simulation applications. J. Med. Eng. Technol. 29(2), 53-63 (2005)

12. Obuchowski, N.A.: Receiver operating characteristic curves and their use in radiology. Radiology 229(1), 3-8 (2003)

13. Ellis, H., Moran, B.J., Thompson, J.N., Parker, M.C., Wilson, M.S., Menzies, D., McGuire, A., Lower, A.M., Hawthorn, R.J., O'Brien, F., Buchan, S., Crowe, A.M.: Adhesion-related hospital readmissions after abdominal and pelvic surgery: a retrospective cohort study. Lancet 353(9163), 1476-1480 (1999)

14. Tan, H.L., Shankar, K.R., Ade-Ajayi, N., Guelfand, M., Kiely, E.M., Drake, D.P., De Bruyn, R., McHugh, K., Smith, A.J., Morris, L., Gent, R.: Reduction in visceral slide is a good sign of underlying postoperative viscero-parietal adhesions in children. J. Pediatr. Surg. 38(5), 714-716 (2003) 